\title{
Different approaches to influence based on social networks and simple games
}

\author{
Michel Grabisch and Agnieszka Rusinowska
}

\begin{abstract}
We present an overview of research on a certain model of influence in a social network. Each agent has to make an acceptance/rejection decision, and he has an inclination to choose either the yes-action or the no-action. The agents are embedded in a social network which models influence between them. Due to the influence, a decision of an agent may differ from his preliminary inclination. Such a transformation between the agents' inclinations and their decisions are represented by an influence function. Follower functions encode the players who constantly follow the opinion of a given unanimous coalition. We examine properties of the influence and follower functions and study the relation between them. The model of influence is also compared to the framework of command games in which a simple game is built for each agent. We study the relation between command games and influence functions. We also define influence indices and determine the relations between these indices and some well-known power indices. Furthermore, we enlarge the set of possible yes/no actions to multi-choice games and investigate the analogous tools related to influence in the multi-choice model.
\end{abstract}

Michel Grabisch

Université Paris I Panthéon-Sorbonne, Centre d'Economie de la Sorbonne, 106-112 Bd de l'Hôpital, 75013 Paris, France. e-mail: michel.grabisch@univ-paris1.fr

Agnieszka Rusinowska

GATE, CNRS UMR 5824 - Université Lumière Lyon 2 - Ecole Normale Supérieure LSH, 93, Chemin des Mouilles - B.P.167, 69131 Ecully Cedex, France. e-mail: rusinowska@gate.cnrs.fr 


\section{Introduction}

\subsection{Aim of the paper}

The existence of influence between agents in collective decision-making situations, in which individual agents are to choose among a number of alternatives, may have a considerable impact on the collective decision, and consequently, on the performance of the collective body. Investigating appropriate tools to measure influence is of importance in every organization, at the individual, group and macro levels. The capacity to influence others seems to be as old as the world, since already the biblical story of Adam and Eva can be modeled in terms of influence, and the consequences of this 'first' influence are experienced constantly in the world. Since influence is present practically everywhere, in all kinds of structures where, e.g., personal, social, economic, and political decisions are to be made, it is not surprising that different approaches to the influence issues can be and have been applied. For a short overview of theoretical and empirical studies of political influence and power in groups presented in the political economic literature, we refer, e.g., to van Winden $(2004,[39])$.

The aim of the present paper is to deliver an overview of the key investigations of our research on influence. The model presented in this paper is a game theoretical model and contributes to the literature of cooperative game theory and network theory on interactions and influence between agents. Since there are many worthwhile research on this framework, delivering an overview of research conducted on the influence model is of importance. It helps to realize what has been done on this subject and to which direction it is good to navigate. In the following subsection, we give a short overview of both cooperative and noncooperative approaches to influence, with a particular focus on our own research on this topic.

\subsection{Overview of research on influence}

Already more than fifty years ago, Isbell (1958, [24]) introduced the concept of influence relation to qualitatively compare the a priori influence of voters in a simple game. In a voting game, where players vote either 'yes' or 'no', voter $k$ is said to be at least as influential as voter $j$, if whenever $j$ can transform a losing coalition into a majority by joining it, voter $k$ can achieve the same ceteris paribus. This influence relation is extended in Tchantcho et al. (2008, [38]) to voting games with abstention. Grabisch and Roubens $(1999,[13])$ analyze the concept of interaction among agents. Players in a coalition are said to exhibit a positive (negative) interaction when the worth of the coalition is greater (smaller) than the sum of the individual worths. 
The cooperative game theoretical approach to interaction is also used in $\mathrm{Hu}$ and Shapley (2003a, 2003b, [22, 23]), where the authors apply the command structure of Shapley (Shapley 1994, [36]) to model players' interaction relations by simple games. For each player, boss sets and approval sets are introduced, and based on these sets, a simple game called the command game for a player is built. Given a set of command games, the command function is defined, which assigns to each coalition the set of all players that are 'commandable' by that coalition.

A different approach, related to noncooperative game theory, is applied in Koller and Milch (2003, [25]), where the so called multi-agent influence diagrams are introduced. These diagrams are a graphical representation for noncooperative games, and represent decision problems involving multiple agents.

One of the concepts naturally related to influence is the concept of leadership. DeMarzo (1992, [8]) examines the set of outcomes sustainable by a leader with the power to make suggestions which are important even if players can communicate and form coalitions. Van den Brink et al. (2009, [5]) define the satisfaction and power scores for opinion leaders - followers structures and examine common properties of these scores.

As mentioned in Hojman and Szeidl (2006, [20]), individual decisions and strategic interaction are both embedded in a social network. Social networks are therefore particularly useful in analyzing influence. In Lopez-Pintado (2008, [28]), where the author stresses the fact that decisions of individuals are often influenced by the decisions of other individuals, a network of interacting agents whose actions are determined by the actions of their neighbors is studied.

The point of departure for our research on influence is a framework originally introduced in Hoede and Bakker (1982, [19]). The model concerns influence in a social network in which agents are to make an acceptance/rejection decision on a certain proposal. Each agent has an inclination to say either 'yes' or 'no' on the proposal, but agents may influence the decisions of others, and consequently the agents' decisions may differ from their preliminary inclinations. Such a transformation from the inclinations to the decisions is represented by an influence function. In Hoede and Bakker (1982, [19]) the concept of decisional power (called later in some related papers the Hoede-Bakker index) is introduced. Some properties of this index are studied in Rusinowska and de Swart (2007, [34]), where the authors examine if the Hoede-Bakker index satisfies some postulates for power indices, like the monotonicity postulate, the donation postulate, and the bloc postulate (see e.g., Felsenthal and Machover 1998, [10]), and if the Hoede-Bakker index displays some voting power paradoxes, like the redistribution paradox (Fischer and Schotter 1978, [12]; see also Schotter 1981, [35]), the paradox of new members (Brams 1975, [3]; Brams and Affuso 1976, [4]), and the paradox of large size (Brams 1975, [3]). Following a probabilistic approach to power indices (see e.g., Laruelle and Valenciano 2005, [27]), Rusinowska and de Swart (2006, [33]) investigate 
a generalization of the Hoede-Bakker index that coincides with the Penrose measure (Penrose 1946, [29]; see also Banzhaf 1965, [1]), and some modifications of this index that coincide with other well known power indices, like the Rae index (Rae 1969, [30]), the Coleman indices (Coleman 1971, 1986, $[6,7]$ ), and the König-Bräuninger index (König and Bräuninger 1998, [26]). Analogous modifications of the Hoede-Bakker index to the Shapley-Shubik index (Shapley and Shubik 1954, [37]) and the Holler-Packel index (Holler and Packel 1983, [21]) are presented in Rusinowska (2009, [32]). In Rusinowska (2008, [31]) the not-preference based version of the Hoede-Bakker index is investigated.

As noticed in our first paper on influence (Grabisch and Rusinowska 2009b, [16]), the Hoede-Bakker index does not give a full description of the influence, in the sense that it hides the actual role of the influence function. This observation has initiated our larger project on the model of influence in a social network, with the aim to investigate measures of influence and other tools to deal with this phenomenon. In Grabisch and Rusinowska (2009b, $[16]$ ), the concept of a weighted influence index of a coalition on an individual is defined. We consider different influence functions, like the majority function, the guru function, the identity function, the reversal function, the mass psychology function, and study their properties. In particular, the set of followers, the kernel of an influence function, and a purely influential function are analyzed.

As mentioned above, another framework which models players' interactions is the framework of command games introduced by $\mathrm{Hu}$ and Shapley (2003a, 2003b, [22, 23]). In Grabisch and Rusinowska (2009a, [15]), we study the relation between this framework and the influence model and show that the model of influence is more general than the command games. In particular, we define several influence functions which capture the command structure. Moreover, we propose a more general definition of the influence index and show that under some assumptions several well-known power indices coincide with some expressions of the weighted influence indices.

In Grabisch and Rusinowska (2008, [14]), we study the exact relation between two central concepts of the influence model: the influence function and the follower function. We deliver sufficient and necessary conditions for a function to be a follower function, and describe the structure of the set of all influence functions that lead to a given follower function. Moreover, we investigate the exact relations between the key concepts of the command games and of the influence model. A sufficient and necessary condition for the equivalence between an influence function and a command game is delivered. We also find sufficient and necessary conditions for a function to be a command function, and describe the minimal sets generating a command game.

In Grabisch and Rusinowska (2009d, [18]), the yes/no model is extended to the influence model in which each agent has a totally ordered set of possible actions. The generalized influence indices and other tools related to 
the multi-choice model are investigated and the results are compared to the ones obtained in the yes/no model of influence. In Grabisch and Rusinowska (2009c, [17]), we consider another generalized model of influence in which each player has a continuum of actions.

\subsection{Structure of the paper}

In the remaining parts of the paper, we present a formal description of our investigations on the selected influence issues. Section 2 concerns the yes/no model of influence. We describe the model and recapitulate some results on the key concepts of the influence model. In Section 3 the framework of command games and some of the relations between this framework and the yes/no influence model are presented. In Section 4 we mention the generalized model of influence in which agents have a totally ordered set of possible actions. Section 5 gives some reflections on the problem of identifying the model of influence in a practical situation. Section 6 is devoted to concluding remarks on our future research on the influence issues.

\section{The model of influence in a social network}

\subsection{Description of the model and weighted influence indices}

We consider a social network with a set of agents (players, actors, voters) denoted by $N:=\{1,2, \ldots, n\}$ who are to make a certain acceptance-rejection decision on a specific proposal. Each agent has an inclination either to say 'yes' (denoted by +1 ) or 'no' (denoted by -1 ). By the inclination of an agent we mean an action that the agent would choose being completely 'on his own', that is, without any interaction with other agents and not being influenced by others. Let $i=\left(i_{1}, i_{2}, \ldots, i_{n}\right)$ denote an inclination vector and $I:=\{-1,+1\}^{n}$ be the set of all inclination vectors. For convenience, $(1,1, \ldots, 1) \in I$ and $(-1,-1, \ldots,-1) \in I$ are denoted by $1_{N}$ and $-1_{N}$, respectively, and also for mixed cases like $\left(-1_{N \backslash S}, 1_{S}\right)$.

Agents in such a social network may influence each other, and due to the influences, the final decision of an agent may be different from his original inclination. Formally, each inclination vector $i \in I$ is transformed into a decision vector $B i=\left((B i)_{1},(B i)_{2}, \ldots,(B i)_{n}\right)$, where $B: I \rightarrow I, i \mapsto B i$ is the influence function. The set of all influence functions will be denoted by $\mathcal{B}$.

What do we mean by influence and how is it modeled in our framework? In our most general statement, we say that an agent is influenced if the 
decision of the agent is different from his (original) inclination. In Grabisch and Rusinowska (2009b, [16]) we distinguish between a direct influence and an opposite influence. The direct influence of a unanimous coalition on an agent, that is, a coalition of players with the same inclination, means that the agent's inclination is different from the inclination of that coalition, but his decision coincides with the inclination of the coalition. Under the opposite influence of a coalition on an agent, the inclination of the agent coincides with the inclination of the coalition, but his decision is different from the inclination of the coalition. In the case of direct influence, which is the most common, the agent changes his opinion because he may be convinced by the arguments of the influencing coalition, or for some political, hierarchical or more personal reason, he feels obliged to follow that coalition. On the other hand, the opposite influence is a kind of reactive behavior. The agent, again for some political or personal reason, systematically decides for the opposite opinion of the influencing coalition. In the present paper, we consider for simplicity only the notion of the direct influence, and hence, in all following definitions we will omit the word 'direct'.

Let us introduce several notations for convenience. Cardinality of sets $S, T, \ldots$ will be denoted by the corresponding lower case $s, t, \ldots$ We omit braces for sets, e.g., $\{k, m\}, N \backslash\{j\}, S \cup\{j\}$ will be written $k m, N \backslash j, S \cup j$, etc. For any $S \subseteq N,|S| \geq 2$, we introduce the set $I_{S}$ of all inclination vectors under which all members of $S$ have the same inclination

$$
I_{S}:=\left\{i \in I \mid \forall k, j \in S\left[i_{k}=i_{j}\right]\right\}
$$

and $I_{k}:=I$, for any $k \in N$. By $i_{S}$ we denote the value $i_{k}$ for some $k \in S$, $i \in I_{S}$. Let for each $S \subseteq N$ and $j \in N \backslash S, I_{S \rightarrow j}$ denote the set of all inclination vectors of potential influence of $S$ on $j$, that is,

$$
I_{S \rightarrow j}:=\left\{i \in I_{S} \mid i_{j}=-i_{S}\right\}
$$

and additionally, for each $B \in \mathcal{B}$, let $I_{S \rightarrow j}^{*}(B)$ denote the set of all inclination vectors of observed influence of $S$ on $j$ under $B \in \mathcal{B}$, that is,

$$
I_{S \rightarrow j}^{*}(B):=\left\{i \in I_{S \rightarrow j} \mid(B i)_{j}=i_{S}\right\} .
$$

In Grabisch and Rusinowska (2009b, [16]), we introduce the weighted influence indices, whose main idea is to give a relative importance to the different inclination vectors. For each $S \subseteq N, j \in N \backslash S$ and $i \in I_{S}$, we introduce a weight $\alpha_{i}^{S \rightarrow j} \in[0,1]$ of influence of coalition $S$ on $j \in N \backslash S$ under the inclination vector $i \in I_{S}$. There is no normalization on the weights, but we assume that for each $S \subseteq N$ and $j \in N \backslash S$, there exists $i \in I_{S \rightarrow j}$ such that $\alpha_{i}^{S \rightarrow j}>0$. Moreover, we impose the symmetry assumption that $\alpha_{i}^{S \rightarrow j}$ depends solely on the number of agents having the same inclination as $S$ under $i \in I_{S}$. 
Given $B \in \mathcal{B}$, for each $S \subseteq N, j \in N \backslash S$, the weighted influence index of coalition $S$ on player $j$ is defined as

$$
d_{\alpha}(B, S \rightarrow j):=\frac{\sum_{i \in I_{S \rightarrow j}^{*}(B)} \alpha_{i}^{S \rightarrow j}}{\sum_{i \in I_{S \rightarrow j}} \alpha_{i}^{S \rightarrow j}} \in[0,1] .
$$

It is the (weighted) proportion of situations of observed influence among all situations of potential influence. Two particular ways of weighting lead to the possibility influence index $\bar{d}(B, S \rightarrow j)$, under which any possibility of influence is taken into account, and the certainty influence index $\underline{d}(B, S \rightarrow j)$, where we take into account only the situations in which all agents outside $S \cup j$ have the inclination different from the inclination of $S$. We have for each $S \subseteq N, j \in N \backslash S$ and $B \in \mathcal{B}$

$$
\bar{d}(B, S \rightarrow j)=d_{\bar{\alpha}}(B, S \rightarrow j), \text { where } \bar{\alpha}_{i}^{S \rightarrow j}=1 \text { for each } i \in I_{S}
$$

and

$$
\begin{aligned}
\underline{d}(B, S \rightarrow j) & =d_{\underline{\alpha}}(B, S \rightarrow j), \text { where for each } i \in I_{S} \\
\underline{\alpha}_{i}^{S \rightarrow j} & = \begin{cases}1, & \text { if } \forall p \notin S \cup j, i_{p}=-i_{S} \\
0, & \text { otherwise. }\end{cases}
\end{aligned}
$$

Consequently, we can write

$$
\begin{gathered}
\bar{d}(B, S \rightarrow j)=\frac{\left|I_{S \rightarrow j}^{*}(B)\right|}{\left|I_{S \rightarrow j}\right|} \in[0,1] \\
\underline{d}(B, S \rightarrow j)=\frac{\left|\left\{i \in I_{S \rightarrow j}^{*}(B) \mid \forall p \notin S\left[i_{p}=-i_{S}\right]\right\}\right|}{2} \in\left\{0, \frac{1}{2}, 1\right\} .
\end{gathered}
$$

The possibility influence index gives therefore the fraction of potential influence situations that happen to be situations of observed influence indeed. The certainty influence index measures also such a fraction, except that it focuses only on situations in which the coalition in question is the only one which (directly) influences the agent.

\subsection{Follower functions and influence functions}

The key concept of the influence framework is the concept of follower of a given coalition, that is, an agent who always follows the inclination of that coalition when all members of the coalition have the same inclination. The follower function of $B \in \mathcal{B}$ is a mapping $F_{B}: 2^{N} \rightarrow 2^{N}$ defined as

$$
F_{B}(S):=\left\{k \in N \mid \forall i \in I_{S},(B i)_{k}=i_{S}\right\}, \quad \forall S \subseteq N, S \neq \emptyset
$$


and $F_{B}(\emptyset):=\emptyset$. We say that $F_{B}(S)$ is the set of followers of $S$ under $B$. The set of all follower functions is denoted by $\mathcal{F}$. In Grabisch and Rusinowska (2009b, [16]), it is shown that

$$
d_{\alpha}(B, S \rightarrow j)=1, \quad \forall j \in F_{B}(S) \backslash S .
$$

Another important concept of the influence model is the concept of kernel of an influence function, which is the set of 'truly' influential coalitions. Assume $F_{B}$ is not identically the empty set. The kernel of $B$ is defined as

$$
\mathcal{K}(B):=\left\{S \in 2^{N} \mid F_{B}(S) \neq \emptyset \text {, and } S^{\prime} \subset S \Rightarrow F_{B}\left(S^{\prime}\right)=\emptyset\right\} .
$$

As defined before, in order to model influences between players, that is, to represent a transformation between agents' inclinations and their decisions, an influence function is used. We like to recapitulate definitions and some basic properties of some of the influence functions defined in Grabisch and Rusinowska (2009b, [16]) that model different types of influence. Four functions will be mentioned: the identity function (which models the absence of any influence), the reversal function (which depicts a systematic reversal of inclination), the guru function (which describes following the guru by each agent in every situation, assuming such a guru exists), and the majority function (under which if a majority of agents has the positive inclination, then all agents choose the yes-option, otherwise all agents choose the no-action). Let us recall definitions of these influence functions.

- The identity function $\mathrm{Id} \in \mathcal{B}$ is defined by

$$
\operatorname{ld} i=i, \quad \forall i \in I \text {. }
$$

- The reversal function $-\mathrm{Id} \in \mathcal{B}$ is defined by

$$
(-\mathrm{ld}) i=-i, \quad \forall i \in I \text {. }
$$

- Let $\widetilde{k} \in N$ be a particular player called the guru.

The guru influence function $\operatorname{Gur}^{[\widetilde{k}]} \in \mathcal{B}$ is defined by

$$
\left(\operatorname{Gur}^{[\widetilde{k}]} i\right)_{j}=i_{\widetilde{k}}, \quad \forall i \in I, \quad \forall j \in N .
$$

- Let $n \geq t>\left\lfloor\frac{n}{2}\right\rfloor$, and for any $i \in I$ we define $i^{+}:=\left\{k \in N \mid i_{k}=+1\right\}$. The majority influence function $\mathrm{Maj}^{[t]} \in \mathcal{B}$ is defined by

$$
\operatorname{Maj}^{[t]} i:=\left\{\begin{array}{ll}
1_{N}, & \text { if }\left|i^{+}\right| \geq t \\
-1_{N}, & \text { if }\left|i^{+}\right|<t
\end{array}, \quad \forall i \in I .\right.
$$


In Grabisch and Rusinowska (2009b, [16]), we prove that the follower functions of these influence functions, for each $S \subseteq N$, are equal to

$$
\begin{gathered}
F_{\mathrm{ld}}(S)=S, \quad F_{-\mathrm{ld}}(S)=\emptyset, \quad F_{\mathrm{Gur}[\tilde{k}]}(S)= \begin{cases}N, & \text { if } \widetilde{k} \in S \\
\emptyset, & \text { if } \widetilde{k} \notin S,\end{cases} \\
F_{\mathrm{Maj}^{[}[t]}(S)= \begin{cases}N, & \text { if } s \geq t \\
\emptyset, & \text { if } s<t\end{cases}
\end{gathered}
$$

and the kernels of these influence functions are

$$
\begin{gathered}
\mathcal{K}(\text { Id })=\{\{k\}, k \in N\}, \quad \mathcal{K}(- \text { Id })=\emptyset, \quad \mathcal{K}\left(\operatorname{Gur}^{[\tilde{k}]}\right)=\{\widetilde{k}\}, \\
\mathcal{K}\left(\mathrm{Maj}^{[t]}\right)=\{S \subseteq N|| S \mid=t\} .
\end{gathered}
$$

In Grabisch and Rusinowska (2008, [14]), we establish the exact relation between the influence function and the follower function. In particular, we find sufficient and necessary conditions for a function to be the follower function of some influence function. Moreover, given a follower function, we find the smallest and greatest influence functions that lead to this follower function.

First of all, note that while there is no restriction on an influence function $B: 2^{N} \rightarrow 2^{N}$, any follower function $F_{B}: 2^{N} \rightarrow 2^{N}$ should satisfy some conditions. The mapping $\Phi: \mathcal{B} \rightarrow\left(2^{N}\right)^{\left(2^{N}\right)}$, defined by $B \mapsto \Phi(B):=F_{B}$ is neither a surjection nor an injection (that is, several different $B$ 's may have the same follower function, and there are functions in $\left(2^{N}\right)^{\left(2^{N}\right)}$ which cannot be the follower function of some influence function). We have $\Phi(\mathcal{B})=\mathcal{F}$. In Grabisch and Rusinowska (2008, [14]), we prove that a function $F: 2^{N} \rightarrow 2^{N}$ is a follower function of some $B \in \mathcal{B}$ (i.e., $F_{B}=F$, or $\Phi(B)=F$ ) if and only if it satisfies the following three conditions:

- $F(\emptyset)=\emptyset$;

- $F$ is an isotone function $\left(S \subseteq S^{\prime}\right.$ implies $\left.F(S) \subseteq F\left(S^{\prime}\right)\right)$;

- If $S \cap T=\emptyset$, then $F(S) \cap F(T)=\emptyset$.

Moreover, the smallest and greatest influence functions belonging to $\Phi^{-1}(F)$ are respectively the influence functions $\underline{B}_{F}$ and $\bar{B}_{F}$, defined by, for all $i \in I$ and all $k \in N$ :

$$
\begin{aligned}
& \left(\underline{B}_{F} i\right)_{k}:=\left\{\begin{array}{ll}
+1, & \text { if } k \in F\left(S^{+}(i)\right) \\
-1, & \text { otherwise }
\end{array},\right. \\
& \left(\bar{B}_{F} i\right)_{k}:= \begin{cases}-1, & \text { if } k \in F\left(S^{-}(i)\right) \\
+1, & \text { otherwise }\end{cases}
\end{aligned}
$$

where $S^{ \pm}(i):=\left\{j \in N \mid i_{j}= \pm 1\right\}$.

For instance, if $F(S)=\emptyset$ for all $S \subseteq N$, then $\underline{B} \equiv-1_{N}$ and $\bar{B} \equiv 1_{N}$. If $F=\mathrm{Id}$, then $\Phi^{-1}(\mathrm{Id})=\{\mathrm{Id}\}$. 
In Grabisch and Rusinowska (2008, [14]), we also find the (algebraic) structure of $\Phi^{-1}(F)$, i.e., the set of all influence functions that lead to the follower function $F$ and we indicate how to compute it. This structure happens to be a distributive lattice.

\subsection{Example}

In order to illustrate the concepts introduced in the previous subsections, let us consider a three-agent network, i.e., $N=\{1,2,3\}$, with the following principles of the decision-making process:

(i) Agent 1 follows himself;

(ii) Agent 2 follows agent 1;

(iii) Agent 3 follows the majority (i.e., he decides according to the inclination of at least two agents).

Figure 1 shows a social network for this example. An arc from player $j$ to $k$ means that $j$ influences player $k$.

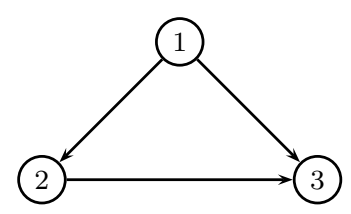

Fig. 1 Three-agent social network

The set of all inclination vectors is $I=\{-1,+1\}^{3},|I|=8$. Table 1 presents the influence function $B$ for the example. Please note that $B$ is a kind of a mixture of the three influence functions mentioned in Section 2.2: agent 1 uses the identity function Id, agent 2 decides according to his guru $\widetilde{k}=1$, and agent 3 applies the majority function Maj ${ }^{[t]}$ with $t=2$.

$$
\begin{array}{rl||c|c|c|c|c|}
i \in I & (1,1,1) & (1,1,-1) & (1,-1,1) & (-1,1,1) & (1,-1,-1) & (-1,1,-1) \\
\hline B i & (1,1,1) & (1,1,1) & (1,1,1) & (-1,-1,1) & (1,1,-1) & (-1,-1,-1) \\
5 & \multicolumn{3}{c|}{i \in I} & (-1,-1,1) & (-1,-1,-1) \\
\hline B i & (-1,-1,-1) & (-1,-1,-1)
\end{array}
$$

Table 1 The influence function $B$

Table 2 shows the set of followers under $B$ of each coalition. Obviously, the conditions for a follower function recapitulated in Section 2.2 are satisfied by $F_{B}$. 


\begin{tabular}{c||c|c|c|c|c|c|c|c}
$S \subseteq N$ & $\emptyset$ & 1 & 2 & 3 & 12 & 13 & 23 & $N$ \\
\hline$F_{B}(S)$ & $\emptyset$ & 12 & $\emptyset$ & $\emptyset$ & $N$ & $N$ & 3 & $N$
\end{tabular}

Table 2 The sets of followers under $B$

From Table 2 we get the kernel of the influence function $B$

$$
\mathcal{K}(B)=\{1,23\}
$$

and using Table 1, we can calculate the influence indices. Note that for each weighted influence index, we have

$$
d_{\alpha}(B, 1 \rightarrow 2)=d_{\alpha}(B, 13 \rightarrow 2)=d_{\alpha}(B, 12 \rightarrow 3)=1
$$

which illustrates the property of the weighted influence index mentioned in Section 2.2, and moreover

$$
d_{\alpha}(B, 2 \rightarrow 1)=d_{\alpha}(B, 3 \rightarrow 1)=d_{\alpha}(B, 23 \rightarrow 1)=0 .
$$

Table 3 presents the possibility and certainty influence indices $(\bar{d}(B, S \rightarrow j), \underline{d}(B, S \rightarrow j))$ for each $\emptyset \neq S \subset N$ and $j \in N \backslash S$.

\begin{tabular}{c||c|c|c|c|c|c}
\multicolumn{1}{c||}{$\rightarrow$} & 1 & 2 & 3 & 12 & 13 & 23 \\
\hline$\downarrow \downarrow$ & & & & & & \\
\hline 1 & - & $(0,0)$ & $(0,0)$ & - & - & $(0,0)$ \\
\hline 2 & $(1,1)$ & - & $\left(\frac{1}{2}, 0\right)$ & - & $(1,1)$ & - \\
\hline 3 & $\left(\frac{1}{2}, 0\right)$ & $\left(\frac{1}{2}, 0\right)$ & - & $(1,1)$ & - & -
\end{tabular}

Table 3 The possibility and certainty influence indices $(\bar{d}(B, S \rightarrow j), \underline{d}(B, S \rightarrow j))$

Note that in this example the certainty influence index is either 0 or 1 , but is never equal to $\frac{1}{2}$. This is related to the neutrality of the influence function $B$ defined in Table 1 which states that $B(-i)=-B i$ for each $i \in I$.

\section{The command games}

\subsection{Command games and command functions}

The framework of command games has been introduced in $\mathrm{Hu}$ and Shapley (2003a, 2003b, [22, 23]), and later analyzed in Grabisch and Rusinowska $(2008,2009 a,[14,15])$. Let us recall the key concepts of this model. Let $N=\{1, \ldots, n\}$ be the set of players. For $k \in N$ and $S \subseteq N \backslash k$ : 
- $S$ is a boss set for $k$ if $S$ determines the choice of $k$;

- $S$ is an approval set for $k$ if $k$ can act with an approval of $S$.

For each $k \in N$, a simple game $\left(N, \mathcal{W}_{k}\right)$ is built, called the command game for $k$, with the set of winning coalitions defined by

$\mathcal{W}_{k}:=\{S \mid S$ is a boss set for $k\} \cup\{S \cup k \mid S$ is a boss or approval set for $k\}$.

The boss and approval sets for $k$ can be recovered by

$$
\begin{gathered}
\text { Boss }_{k}=\left\{S \subseteq N \backslash k \mid S \in \mathcal{W}_{k}\right\}=\mathcal{W}_{k} \cap 2^{N \backslash k} \\
\operatorname{App}_{k}=\left\{S \subseteq N \backslash k \mid S \cup k \in \mathcal{W}_{k} \text { but } S \notin \mathcal{W}_{k}\right\} .
\end{gathered}
$$

We have Boss $_{k} \cap \mathrm{App}_{k}=\emptyset$. In particular, if $\mathrm{App}_{k}=2^{N \backslash k}$, then $k$ is called a free agent, since he needs no approval $\left(\emptyset \in \mathrm{App}_{k}\right)$ and nobody can boss him Boss $_{k}=\emptyset$ ). If $\mathrm{App}_{k}=\emptyset$, then $k$ is called a $\operatorname{cog}$.

Given a set of command games $\left\{\left(N, \mathcal{W}_{k}\right), k \in N\right\}$, the command function $\omega: 2^{N} \rightarrow 2^{N}$ is defined as

$$
\omega(S):=\left\{k \in N \mid S \in \mathcal{W}_{k}\right\}, \forall S \subseteq N .
$$

$\omega(S)$ is the set of all members that are 'commandable' by $S$.

As noticed in Grabisch and Rusinowska (2008, [14]), any set of command games $\left\{\left(N, \mathcal{W}_{k}\right), k \in N\right\}$ can be viewed as a mapping $\Omega: N \times 2^{N} \rightarrow\{0,1\}$, with

$$
(k, S) \mapsto \Omega(k, S)=\left\{\begin{array}{ll}
1, & \text { if } S \in \mathcal{W}_{k} \\
0, & \text { otherwise }
\end{array} .\right.
$$

Recall that for any $S \subseteq N$, the principal filter of $S$ is defined as $\uparrow S:=$ $\{T \subseteq N \mid T \supseteq S\}$. A normal command game $\Omega$ is a set of simple games $\left\{\left(N, \mathcal{W}_{k}\right), k \in N\right\}$ satisfying the two conditions:

- For each $k \in N$, there exists a minimal nonempty family of nonempty subsets $S_{1}^{k}, \ldots, S_{l_{k}}^{k}$ (called the generating family of $\mathcal{W}_{k}$ ) such that $\mathcal{W}_{k}=\uparrow S_{1}^{k} \cup \ldots \cup \uparrow S_{l_{k}}^{k}$.

- For each $k \in N, S_{1}^{k} \cap \cdots \cap S_{l_{k}}^{k} \neq \emptyset$.

The last condition is motivated by the following fact: if there exist two disjoint boss sets for agent $k$, then there will be a conflict if the boss sets have a different opinion. We denote by $\mathcal{G}$ the set of all normal command games. There exists a bijection $\Psi: 2^{N \times 2^{N}} \rightarrow\left(2^{N}\right)^{\left(2^{N}\right)}$ defined by

$$
\begin{gathered}
\Psi(\Omega)=\omega, \quad \text { with } \omega(S):=\{k \in N \mid \Omega(k, S)=1\}, \quad \forall S \subseteq N \\
\Psi^{-1}(\omega)=\Omega, \quad \text { with } \Omega(k, S)=1 \text { iff } k \in \omega(S) .
\end{gathered}
$$

In Grabisch and Rusinowska (2008, [14]), we show the exact relation between command games and command functions. We prove that $\omega \in\left(2^{N}\right)^{\left(2^{N}\right)}$ 
corresponds to some normal command game, i.e., $\omega \in \Psi(\mathcal{G})$, if and only if the following conditions are satisfied:

- $\omega(\emptyset)=\emptyset, \omega(N)=N$

- $\omega$ is isotone;

- If $S \cap S^{\prime}=\emptyset$, then $\omega(S) \cap \omega\left(S^{\prime}\right)=\emptyset$.

Note the similarity between the sufficient and necessary conditions for a function to be the command function of some command game, and those for a function to be the follower function of some influence function. We can conclude that comparing the command framework with the influence model should be based on comparing command games with influence functions, and will be closely related to the relation between command functions and follower functions.

\subsection{Command games and influence functions}

We investigate the relation between command games and influence functions. In particular, we are interested in equivalence between command games and influence functions. An influence function $B$ and a command game $\Omega$ are said to be equivalent if $\omega \equiv F_{B}$.

In Grabisch and Rusinowska (2008, [14]), we show that if $B$ is an influence function, then there exists a unique normal command game $\Omega$ equivalent to $B$ if and only if $F_{B}(N)=N$. Moreover, if $\Omega$ is a normal command game, then any influence function in $\Phi^{-1}(\omega)$ is equivalent to $\Omega$.

Let us present command games equivalent to the four influence functions recapitulated in Section 2.2. In Grabisch and Rusinowska (2009a, [15]), we prove the following:

- The identity function $\mathrm{Id} \in \mathcal{B}$ is equivalent to the set of command games $\left\{\left(N, \mathcal{W}_{k}^{\text {ld }}\right) \mid k \in N\right\}$, where

$$
\mathcal{W}_{k}^{\mathrm{ld}}=\{S \subseteq N \mid k \in S\}, \forall k \in N .
$$

- There is no set of command games equivalent to the reversal function - Id $\in \mathcal{B}$.

- The guru function Gur ${ }^{[\widetilde{k}]} \in \mathcal{B}$ is equivalent to the set of command games $\left\{\left(N, \mathcal{W}_{k}^{\text {Gur }^{[\tilde{k}]}}\right) \mid k \in N\right\}$, where

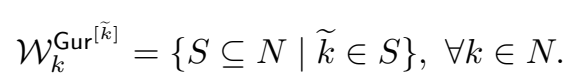

- The majority function $\mathrm{Maj}^{[t]} \in \mathcal{B}$, where $n \geq t>\left\lfloor\frac{n}{2}\right\rfloor$, is equivalent to the set of command games $\left\{\left(N, \mathcal{W}_{k}^{\mathrm{Maj}^{[t]}}\right) \mid k \in N\right\}$ with 


$$
\mathcal{W}_{k}^{\text {Maj }^{[t]}}=\{S \subseteq N \mid s \geq t\}, \forall k \in N
$$

Note that the existence of a unique normal command game equivalent to $B \in\left\{\mathrm{Id}\right.$, Gur $^{[\tilde{k}]}$, Maj $\left.^{[t]}\right\}$ could be already concluded from Section 2.2, where it is straightforward to see that $F_{B}(N)=N$ for $B \in\left\{\mathrm{ld}, \operatorname{Gur}^{[\tilde{k}]}, \mathrm{Maj}^{[t]}\right\}$. On the other hand, we note that $F_{-\mathrm{ld}}(N)=\emptyset$.

\subsection{Example continued}

We turn to the example presented in Section 2.3, and model it in terms of a command structure. Hence, we have a three-agent game $N=\{1,2,3\}$, in which agent 1 follows himself, agent 2 follows agent 1 , and agent 3 follows the majority. The command game $\Omega$ for the example is defined as follows:

$$
\begin{gathered}
\mathcal{W}_{1}=\mathcal{W}_{2}=\{1,12,13,123\} \\
\mathcal{W}_{3}=\{12,13,23,123\}
\end{gathered}
$$

Hence, we have

$$
\begin{gathered}
\text { Boss }_{1}=\emptyset, \quad \text { Boss }_{2}=\{1,13\}, \quad \text { Boss }_{3}=\{12\} \\
\text { App }_{1}=\{\emptyset, 2,3,23\}, \quad \text { App }_{2}=\emptyset, \quad \text { App }_{3}=\{1,2\} .
\end{gathered}
$$

Note that agent 1 is a free agent, and agent 2 is a cog.

The command function $\omega$ for this example is presented in Table 4 .

\begin{tabular}{c||c|c|c|c|c|c|c|c}
$S \subseteq N$ & $\emptyset$ & 1 & 2 & 3 & 12 & 13 & 23 & $N$ \\
\hline$\omega(S)$ & $\emptyset$ & 12 & $\emptyset$ & $\emptyset$ & $N$ & $N$ & 3 & $N$
\end{tabular}

Table 4 The command function $\omega$

Note that the influence function $B$ defined in Section 2.3 and the command game $\Omega$ of the present example are equivalent, because $\omega \equiv F_{B}$.

\subsection{Power and influence indices}

In Grabisch and Rusinowska (2009a, [15]), a more general version of the weighted influence index is defined which can cover all imaginable types of influence, in particular, the direct influence, the opposite influence, an influence of a coalition on its member, etc. First of all, it is assumed that only a coalition with all members unanimous in inclinations may influence an agent. 
An influence of a coalition $S$ on a player $j$ takes place if $(B i)_{j}=\lambda$, where $\lambda \in\left\{-i_{j}, i_{S},-i_{S},+1,-1\right\}$. Hence, the set $I_{S \rightarrow j, \lambda}(B)$ of all inclination vectors of influence of $S$ on $j$ under $B$ is defined as

$$
I_{S \rightarrow j, \lambda}(B):=\left\{i \in I_{S} \mid(B i)_{j}=\lambda\right\}
$$

and given $B \in \mathcal{B}$, for each $S \subseteq N, j \in N$, the general weighted influence index of coalition $S$ on player $j$ under $B$ is defined as

$$
\psi_{\alpha, \lambda}(B, S \rightarrow j):=\frac{\sum_{i \in I_{S \rightarrow j, \lambda}(B)} \alpha_{i}^{S \rightarrow j}}{\sum_{i \in I_{S}} \alpha_{i}^{S \rightarrow j}} .
$$

In particular, the weighted (direct) influence index defined in the previous section is recovered as

$\psi_{\alpha, \lambda}(B, S \rightarrow j)=d_{\alpha}(B, S \rightarrow j) \quad$ if $\quad \lambda=-i_{j} \quad$ and $\quad \alpha_{i}^{S \rightarrow j}=0 \quad$ for $\quad i_{j}=i_{S}$.

For an arbitrary set of command games, we construct several equivalent (command) influence functions. This shows that the model of influence is broader than the framework of the command games. Moreover, we apply several power indices to the command games and prove that these power indices coincide with some expressions of the general weighted influence indices under the command influence functions. One of the (command) influence function that we define is the influence function with abstention, related to an extended three-action model of influence recapitulated in Section 4.1. In this model, each agent has three options for his decision: 'yes' (denoted by +1 ), 'no' (denoted by -1 ), or 'abstain' (denoted by 0 ), that is, $B(I) \subseteq\{-1,0,+1\}^{n}$.

Given a set of command games $\left\{\left(N, \mathcal{W}_{k}\right) \mid k \in N\right\}$, the command influence function Com is defined for each $k \in N$ and $i \in I$ by

$$
(\operatorname{Com} i)_{k}:= \begin{cases}+1, & \text { if }\left\{j \in N \mid i_{j}=+1\right\} \in \mathcal{W}_{k} \\ -1, & \text { if }\left\{j \in N \mid i_{j}=-1\right\} \in \mathcal{W}_{k} . \\ 0, & \text { otherwise }\end{cases}
$$

According to Com, for each agent $k$ and each inclination vector, if all players with the same inclination forms a winning coalition in his command game, the agent $k$ follows the inclination of this winning coalition. Otherwise, $k$ abstains.

We have shown that for each set of command games $\left\{\left(N, \mathcal{W}_{k}\right) \mid k \in N\right\}$, the command influence function Com is equivalent to this set of command games (in the sense of Section 3.2).

We prove that if $\left\{\left(N, \mathcal{W}_{j}\right) \mid j \in N\right\}$ is a set of command games, and Com is the command influence function defined in (18), then for each $j, k \in N$ 


$$
S h_{k}\left(N, \mathcal{W}_{j}\right)=\psi_{\alpha(S h), \lambda=i_{k}}(\operatorname{Com}, k \rightarrow j)-\psi_{\alpha^{(S h)}, \lambda=-i_{k}}(\operatorname{Com}, k \rightarrow j)
$$

where $S h_{k}\left(N, \mathcal{W}_{j}\right)$ is the Shapley-Shubik index of player $k$ in the command game for $j, \psi_{\alpha(S h), \lambda}$ is the weighted influence index defined in (17), and for each $i \in I$

$$
\alpha_{i}^{(S h) k \rightarrow j}= \begin{cases}\frac{1}{n\left(\begin{array}{c}
n-1 \\
|i|-1
\end{array}\right)}, & \text { if } i_{k}=+1 \\
\frac{1}{n\left(\begin{array}{c}
n-1 \\
n-|i|-1
\end{array}\right)}, & \text { if } i_{k}=-1\end{cases}
$$

and $|i|:=\left|\left\{m \in N \mid i_{m}=+1\right\}\right|$. Moreover,

$$
B z_{k}\left(N, \mathcal{W}_{j}\right)=\psi_{\alpha^{(B z)}, \lambda=i_{k}}(\operatorname{Com}, k \rightarrow j)-\psi_{\alpha^{(B z)}, \lambda=-i_{k}}(\operatorname{Com}, k \rightarrow j)
$$

where $B z_{k}\left(N, \mathcal{W}_{j}\right)$ is the Banzhaf index of player $k$ in the command game for $j, \psi_{\alpha^{(B z), \lambda}}$ is the weighted influence index defined in (17) with

$$
\alpha_{i}^{(B z) k \rightarrow j}=1, \forall i \in I .
$$

This means that both the Shapley-Shubik index and the Banzhaf index of player $k$ in the command game for $j$ are equal to the difference between the weighted influence index in which agent $j$ is said to be influenced by $k$ if he follows $k$, and the weighted influence index in which the influence of $k$ on $j$ means that agent $j$ 's decision is opposite to the inclination of $k$. Both weighted influence indices are measured under the command influence function Com. The difference between the results on these power indices lies only in the weights: while for the Shapley-Shubik index the weights are defined in (19), the weights for the Banzhaf index are always equal to 1.

\section{Enlarging the set of possible yes/no actions}

\subsection{The influence model with an ordered set of possible actions}

In Grabisch and Rusinowska (2009d, [18]), we extend the yes/no model of influence to the framework in which each agent has a totally ordered set of possible actions. We recapitulate in this section the main concepts and results of this work. We investigate the generalized influence indices and other tools related to the multi-choice model.

Let us recapitulate a simplified version of this model. We consider a social network with the set of agents denoted by $N=\{1, \ldots, n\}$. There is a totally ordered (finite) set of possible actions denoted by $\mathcal{A}$. A real number is assigned to each action in $\mathcal{A}$, so that the ordering of these numbers reflect the ordering of the actions (ordinal scale). Let $A$ denote the set of these numbers. 
Assuming there are no two actions with the same rank, we have a bijection between $\mathcal{A}$ and $A$, so that we can deal only with $A$. A particular example is to allow abstention (see, e.g., Braham and Steffen 2002, [2], Felsenthal and Machover 1997, 2001, [9, 11]), and to consider the yes/no-abstention model of influence with $A=\{-1,0,+1\}$, with 0 denoting the action 'to abstain'. The yes/no model of influence considered in the previous sections is obviously covered by this generalized framework with $A=\{-1,+1\}$.

Each player has an inclination to choose one of the actions. Let $i$ denote an inclination vector and $I=A^{n}$ be the set of all inclination vectors ${ }^{1}$. As in the yes/no model, it is assumed that agents may influence each others, and due to the influences, the final decision of a player may be different from his original inclination. Let $B: I \rightarrow I, i \mapsto B i$ denote the influence function, and $B i$ a decision vector. The set of all influence functions will be denoted by $\mathcal{B}$. We introduce for any $\emptyset \neq S \subseteq N$

$$
I_{S}:=\left\{i \in I \mid \forall k, j \in S\left[i_{k}=i_{j}\right]\right\}
$$

which is the set of inclination vectors under which all players in $S$ have the inclination to choose the same action.

We analyze positive influence which measures how much a coalition pulls the agent's decision closer to the inclination of the coalition. A player who has an inclination different from the inclination of a given coalition is said to be influenced by this coalition if his decision is closer to the inclination of the coalition than his inclination was. A direct influence in the yes/no model is therefore a particular case of positive influence. We also investigate negative influence. For each inclination vector in which the members of a given coalition have the same inclination, there is one (or two) action(s) which is (are) the most extreme action(s). These actions lie farthest from the inclination of the coalition. If the inclination of a player is different from such actions, and his decision comes 'closer' to the extreme action, we say that there is a negative influence of the coalition on the player. An opposite influence in the yes/no model is a particular case of negative influence. In the present paper we recapitulate only the positive influence. Let for each $S \subseteq N$ and $j \in N \backslash S$

$$
I_{S \rightarrow j}:=\left\{i \in I_{S} \mid i_{j} \neq i_{S}\right\}
$$

denotes the set of all inclination vectors of potential positive influence of $S$ on $j$. Given coalition $S \subset N$, agent $j \in N \backslash S$, and inclination vector $i \in I_{S \rightarrow j}$, there is a certain distance $\left|i_{j}-i_{S}\right|$ between $i_{j}$ and $i_{S}$. Under the influence, the decision $(B i)_{j}$ of agent $j$ may be different from his inclination, and we can also measure the distance $\left|(B i)_{j}-i_{S}\right|$ between the decision of the agent and the inclination of the coalition. For each $S \subseteq N, j \in N \backslash S$, and $B \in \mathcal{B}$, we define the set of all inclination vectors of influence of $S$ on $j$ under $B$ as

\footnotetext{
${ }^{1}$ We keep for the set of inclination vectors the same notation as in the yes/no model. This should cause no confusion.
} 


$$
I_{S \rightarrow j}^{*}(B):=\left\{i \in I_{S \rightarrow j}||(B i)_{j}-i_{S}|<| i_{j}-i_{S} \mid\right\} .
$$

For each $S \subseteq N, j \in N \backslash S$ and $i \in I_{S \rightarrow j}$, we introduce a weight $\alpha_{i}^{S \rightarrow j} \in$ $[0,1]$ of influence of coalition $S$ on $j \in N \backslash S$ under the inclination vector $i \in I_{S \rightarrow j}$. We assume that for each $S \subseteq N$ and $j \in N \backslash S$, there exists $i \in I_{S \rightarrow j}$ such that $\alpha_{i}^{S \rightarrow j}>0$.

Given $B \in \mathcal{B}$, for each $S \subseteq N, j \in N \backslash S$, the generalized weighted influence index of coalition $S$ on agent $j$ is defined as

$$
D_{\alpha}(B, S \rightarrow j):=\frac{\sum_{i \in I_{S \rightarrow j}^{*}(B)}\left[\left|i_{j}-i_{S}\right|-\left|(B i)_{j}-i_{S}\right|\right] \alpha_{i}^{S \rightarrow j}}{\sum_{i \in I_{S \rightarrow j}}\left|i_{j}-i_{S}\right| \alpha_{i}^{S \rightarrow j}} \in[0,1] .
$$

We can recover the generalized possibility influence index of coalition $S$ on player $j$ as

$$
\bar{D}(B, S \rightarrow j)=D_{\bar{\alpha}}(B, S \rightarrow j), \text { where } \bar{\alpha}_{i}^{S \rightarrow j}=1 \text { for each } i \in I_{S \rightarrow j}
$$

that is,

$$
\bar{D}(B, S \rightarrow j)=\frac{\sum_{i \in I_{S \rightarrow j}^{*}(B)}\left[\left|i_{j}-i_{S}\right|-\left|(B i)_{j}-i_{S}\right|\right]}{\sum_{i \in I_{S \rightarrow j}}\left|i_{j}-i_{S}\right|} .
$$

A follower of a coalition in the generalized influence model is defined as an agent whose decision is never farther from the inclination of the coalition than his inclination was. An agent who always decides according to the inclination of the coalition in question is called a perfect follower of that coalition. Formally, the follower function of $B \in \mathcal{B}$ is a mapping $F_{B}: 2^{N} \rightarrow 2^{N}$ defined as

$$
\begin{aligned}
F_{B(S)}:=\left\{j \in N \mid \forall i \in I_{S}\left[\left[i_{j} \neq i_{S} \Rightarrow\right.\right.\right. & \left.\left|(B i)_{j}-i_{S}\right|<\left|i_{j}-i_{S}\right|\right] \\
& \left.\left.\wedge\left[i_{j}=i_{S} \Rightarrow(B i)_{j}=i_{S}\right]\right]\right\},
\end{aligned}
$$

where $F_{B}(\emptyset):=\emptyset$, and the perfect follower function $F_{B}^{\text {per }}: 2^{N} \rightarrow 2^{N}$ is defined as

$$
F_{B}^{p e r}(S):=\left\{j \in N \mid \forall i \in I_{S}\left[(B i)_{j}=i_{S}\right]\right\} .
$$

Of course, each perfect follower is also a follower, i.e., for each $B \in \mathcal{B}$ and $S \subseteq N, F_{B}^{p e r}(S) \subseteq F_{B}(S)$.

It is important to note that all the above definitions coincide with the ones of the yes/no model if we put $A=\{-1,+1\}$. We show that some of the properties of the follower function in the yes/no model remain valid also in the extended model of influence. In particular,

- $F_{B}$ is an isotone function;

- $F_{B}(S) \cap F_{B}(T)=\emptyset$ whenever $S \cap T=\emptyset$;

- $D_{\alpha}(B, S \rightarrow j)=1$ for each $j \in F_{B}^{\text {per }}(S) \backslash S$. 
In the yes/no model, the last property is satisfied for the set of followers $F_{B}(S)$, but in the multi-choice game it remains valid only for the set of perfect followers $F_{B}^{\text {per }}(S)$.

Assume $F_{B}$ is not identically the empty set. The kernel of $B$ is defined similarly as in the yes/no model of influence, i.e.,

$$
\mathcal{K}(B):=\left\{S \in 2^{N} \mid F_{B}(S) \neq \emptyset \text {, and } S^{\prime} \subset S \Rightarrow F_{B}\left(S^{\prime}\right)=\emptyset\right\} .
$$

Next, we generalize several influence functions $B \in \mathcal{B}$ (defined in the yes/no model) for the multi-choice framework. We investigate the properties of these functions and compare them with the results on the analogous functions in the yes/no model. Let us recapitulate the majority influence function $\widetilde{M a j^{[t]}}$ defined in the extended model, which differs from the majority function $\mathrm{Maj}^{[t]}$ presented in Section 2.

Let $n \geq t>\left\lfloor\frac{n}{2}\right\rfloor$, and introduce for any $i \in I$ and $a \in A$, the set

$$
i^{a}:=\left\{k \in N \mid i_{k}=a\right\} .
$$

The majority influence function $\widetilde{\mathrm{Maj}^{[t]}} \in \mathcal{B}$ is defined by

$$
\left(\widetilde{\operatorname{Maj}^{[t]}} i\right)_{j}:=\left\{\begin{array}{ll}
a, & \text { if } \exists a \in A\left[\left|i^{a}\right| \geq t\right] \\
i_{j}, & \text { otherwise }
\end{array}, \quad \forall i \in I, \quad \forall j \in N .\right.
$$

If a majority of players have an inclination $a$, then all agents decide for $a$, and if not, then each agent decides according to his own inclination.

We prove that the follower function of this majority function, for each $S \subseteq N$, is equal to

$$
F_{\widetilde{\mathrm{Maj}^{j}[t]}}(S)= \begin{cases}N, & \text { if } s \geq t \\ S, & \text { if } n-t<s<t \\ \emptyset, & \text { if } s \leq n-t\end{cases}
$$

and the kernel is $\mathcal{\mathcal { K }}\left(\widetilde{\mathrm{Maj}^{[t]}}\right)=\{S \subseteq N|| S \mid=n-t+1\}$. The results on the set of followers and the kernel for the majority function in the multi-choice model are different from the ones obtained for the yes/no model of influence, which is rather not surprising, since the definitions of the majority influence function in the two models differ from each other. 


\subsection{Example continued}

We turn again to the example presented in Section 2.3, i.e., $N=\{1,2,3\}$, but assume that the agents have a third option to choose, the abstention. We have $A=\{-1,0,+1\}$, and there are 27 possible inclination vectors, $|I|=27$.

For all inclination vectors with inclinations 'yes' or 'no', the extended influence function $B$ in the 3 -action model coincides with the influence function $B$ presented in Section 2.3. We have still to define $B$ when at least one agent has the inclination to abstain. Roughly speaking, in this example we assume the abstention to weaken the influence and independence of agent 1 and to increase the independence of agents 2 and 3. For instance, if agent 1 is inclined to abstain, and the remaining agents are unanimous, he will decide according to their inclination. Agent 2 follows agent 1 except for the situations where agent 1 is inclined to abstain, and agents 2 and 3 are unanimous. When there is at least one 'abstention', agent 3 will follow himself, except the situations when he is inclined to abstain, and the remaining agents are unanimous. In this case agent 3 will decide according to their inclination. Table 5 shows the influence function $B$.

\begin{tabular}{c|c||c|c||c|c}
$i \in I$ & $B i$ & $i \in I$ & $B i$ & $i \in I$ & $B i$ \\
\hline \hline$(-1,-1,-1)$ & $(-1,-1,-1)$ & $(0,0,0)$ & $(0,0,0)$ & $(1,1,1)$ & $(1,1,1)$ \\
\hline$(-1,-1,0)$ & $(-1,-1,-1)$ & $(0,0,-1)$ & $(0,0,-1)$ & $(1,1,-1)$ & $(1,1,1)$ \\
\hline$(-1,0,-1)$ & $(-1,-1,-1)$ & $(0,-1,0)$ & $(0,0,0)$ & $(1,-1,1)$ & $(1,1,1)$ \\
\hline$(0,-1,-1)$ & $(-1,-1,-1)$ & $(-1,0,0)$ & $(-1,-1,0)$ & $(-1,1,1)$ & $(-1,-1,1)$ \\
\hline$(-1,-1,1)$ & $(-1,-1,-1)$ & $(0,0,1)$ & $(0,0,1)$ & $(1,1,0)$ & $(1,1,1)$ \\
\hline$(-1,1,-1)$ & $(-1,-1,-1)$ & $(0,1,0)$ & $(0,0,0)$ & $(1,0,1)$ & $(1,1,1)$ \\
\hline$(1,-1,-1)$ & $(1,1,-1)$ & $(1,0,0)$ & $(1,1,0)$ & $(0,1,1)$ & $(1,1,1)$ \\
\hline$(-1,0,1)$ & $(-1,-1,1)$ & $(0,-1,1)$ & $(0,0,1)$ & $(1,-1,0)$ & $(1,1,0)$ \\
\hline$(-1,1,0)$ & $(-1,-1,0)$ & $(0,1,-1)$ & $(0,0,-1)$ & $(1,0,-1)$ & $(1,1,-1)$
\end{tabular}

Table 5 The influence function $B$

Table 6 presents the generalized possibility influence indices. Note that, for instance, the cases $B(0,-1,1)=(0,0,1)$ and $B(0,1,-1)=(0,0,1)$ count for the influence of agent 3 on agent 2 , since the distance between the inclinations of agents 2 and 3 was 2 , and the distance between the decision of agent 2 and the inclination of agent 3 becomes 1 .

Indeed, when comparing Tables 3 and 6 , we can see that in the 3 -action model the influence indices of agent 1 on agents 2 and 3 , and the influence index of coalition $\{1,2\}$ on agent 3 decreased, while the influence of coalition $\{1,3\}$ on agent 2 is still equal to 1 . The influence of coalition $\{2,3\}$ on agent 1 increased.

Finally, we calculate the sets of followers under $B$ of each coalition. They are presented in Table 7. 


\begin{tabular}{c||c|c|c|c|c|c}
$\begin{array}{r}S \downarrow \\
j\end{array}$ & 1 & 2 & 3 & 12 & 13 & 23 \\
\hline 1 & - & $\frac{1}{9}$ & $\frac{1}{9}$ & - & - & $\frac{1}{3}$ \\
\hline 2 & $\frac{8}{9}$ & - & $\frac{4}{9}$ & - & 1 & - \\
\hline 3 & $\frac{2}{9}$ & $\frac{2}{9}$ & - & $\frac{2}{3}$ & - & -
\end{tabular}

Table 6 The generalized possibility influence indices $\bar{D}(B, S \rightarrow j)$

\begin{tabular}{c||c|c|c|c|c|c|c|c}
$S \subseteq N$ & $\emptyset$ & 1 & 2 & 3 & 12 & 13 & 23 & $N$ \\
\hline$F_{B}(S)$ & $\emptyset$ & $\emptyset$ & $\emptyset$ & $\emptyset$ & 12 & $N$ & 3 & $N$
\end{tabular}

Table 7 The sets of followers under $B$

One can see from comparing Tables 2 and 7 that agent 1 as well as coalition $\{1,2\}$ lost (some of) their followers when allowing the abstention. Moreover, the kernel of the influence function $B$ contains all two-agent coalitions, that is,

$$
\mathcal{K}(B)=\{12,13,23\}
$$

From the comparison of $\mathcal{K}(B)$ in the yes/no model and in the three-action model, we can see that when extending the model to the three-action framework, coalition $\{2,3\}$ remains the 'truly' influential coalition, while agent 1 is not 'truly' influential on his own anymore, but he needs one of the remaining agents to belong to the kernel.

Our conclusions on influence indices, followers, and kernels, are obviously valid only for this example, when we specifically 'weaken' the position of agent 1 by allowing the abstention. However, we like to stress the fact that for an arbitrary example one can apply the same approach and draw conclusions on the impact of enlarging the set of possible actions on agents' influence position.

\subsection{The influence model with a continuum of actions}

In Grabisch and Rusinowska (2009c, [17]), we consider another generalized model of influence in which each player has a continuum of actions. The set of actions is assumed to be a real interval $[a, b]$. Each player has an inclination to choose one of the actions, i.e., by the inclination of a player we mean the particular action from $[a, b]$ the player wants to choose. For the continuum 
case, we have defined and studied, in particular, the influence index of a coalition on a player, several influence functions, the set of followers and perfect followers, and the kernel of an influence function. The main difference between the two generalized models of influence lies naturally in the definitions of the influence indices. While in the previous model (i.e., the model with a totally ordered set of actions), the influence index has been defined by the sums of some expressions over the particular sets, in the continuum case the sums are replaced by integrals. These integrals are calculated over particular sets of inclination vectors which are of a smaller dimension than the set of $n$-inclination vectors. We show the equivalence between the influence index of a coalition on a player and the corresponding influence index in which the coalition in question is treated as one player. For a more detailed analysis of this model we refer to Grabisch and Rusinowska (2009c, [17]).

\section{Levels of knowledge and the identification problem}

Let us come back for simplicity to the yes/no model. So far we have taken for granted that the function $B$ is known, so that influence indices, the follower function, kernels, command games, etc., can be computed. In practice this is too strong an assumption, since the knowledge of $B$ requires the observation of $n 2^{n}$ values, which are 0 or 1 . Specifically, supposing that the inclination vector $i$ is known (there are $2^{n}$ different such vectors), we observe the final decision of each agent ( $n$ values).

Let us first try to establish a kind of hierarchy of knowledge. At the top level of this hierarchy lies the influence function $B$. Its complete definition requires $n 2^{n}$ binary values, and these values are free, i.e., we actually have $n 2^{n}$ degrees of freedom. Knowing $B$ permits to compute all quantities defined in this paper. At the second level lie follower functions. They also require $n 2^{n}$ binary values to be defined, but due to properties that characterize follower functions (see Section 2.2), there are less than $n 2^{n}$ degrees of freedom. In fact, this point is completely elucidated since we know all possible influence functions that give rise to a given follower function: they are given by the function $\Phi^{-1}$ (see Section 2.2). Now, due to the equivalence between command functions of command games and follower functions (up to the condition that $F(N)=N$ ), and moreover between command functions and boss sets and approval sets (see Section 3.1), we can say that the knowledge of boss sets and approval sets lies on the same second level as follower functions.

On the next third level we presumably find indices of influence, although their exact position with respect to follower functions is not known, nor is known their exact relation with influence functions (similarly as in the case of follower functions, one would like to know the set of influence functions giving rise to a given set of values for the influence indices). The number of different values for the influence indices is equal to the number of $S \rightarrow j$, for 
$S \subseteq N, S \neq \emptyset, N$, and $j \notin S$. This gives

$$
\sum_{S \subseteq N S \neq \emptyset, N}(n-s)=\left(2^{n}-2\right) n-\sum_{s=1}^{n-1} s\left(\begin{array}{l}
n \\
s
\end{array}\right)<n 2^{n} .
$$

Therefore, the knowledge of the indices does not permit to recover $B$.

On the fourth level we find influence graphs (social networks), as the one given to explain the example in Section 2.3. An influence graph is only a qualitative description of influence among agents, and does not specify what happens in case of conflict (i.e., when two arrows arrive on the same agent), nor it permits to distinguish between the influences when the inclination of influencing agents is 'yes' or 'no'. The kernel is another type of qualitative information, but which does not seem comparable to influence graphs. The kernel identifies the minimal influencing coalitions, but does not tell whom they influence.

Let us assume that in some experimental situation, observing the behavior of agents, the ultimate knowledge we would like to get is the influence function $B$. However, we can only know it partly, hence what can we do in order to have the most complete possible knowledge on it? We give some thoughts on this problem below.

A first general remark is that we are concerned here with the vast area of machine learning (and therefore optimization, interpolation), and also hypothesis testing and the theory of estimation. We use the generic term of identification for obtaining the complete definition of some function or quantity, as for example the influence function. In any problem of identification, it is important to define what should be done in case of lack of knowledge. In our context, the answer for this is fairly obvious: if there is no observation of influence, by invoking the principle of insufficient reason, just say there is no influence, and therefore $B=\mathrm{Id}$. This defines the general philosophy one should take: use the available knowledge of any kind to construct $B$, and for the regions where $B$ is not known, just put $B=\mathrm{Id}$. The same holds for any kind of notions so far introduced: in case of absence of observation/knowledge, the follower function is identical to the empty set, all influence indices are 0 , etc.

What can we observe in an experimental situation? The direct observation of inclinations and decisions of agents defines $B$ on a small part of its domain. However, a priori information can be used to complete the model, for instance:

(i) It is likely (or well known, often observed, etc.) that coalition $S$ strongly influences agents $j, k, \ldots$;

(ii) We suppose that the underlying model is of the majority type, or of the guru type where agent $j$ plays the rôle of the guru, or of the mass psychology type, etc.

In the first case, we use the mathematical properties underlying our concepts to find the set of functions $B$ compatible with the information we have 
on the followers, degrees, and so on (inverse function problem, completely solved for the case of follower functions). In the second case, the procedure is quite different since this is typically a problem of hypothesis testing and estimation, and therefore the appropriate statistical tools should be used. For example: "Is player $k$ a guru?" is a hypothesis, and an appropriate test should be defined for this. Now, "Is the model of the majority type?" is both a hypothesis testing (yes or no) and an estimation problem, since the threshold $t$ has to be measured. For each hypothesis, there should exist a minimal subset $I_{0} \subseteq I$ of all inclination vectors, so that, if observing decisions for this set $I_{0}$, the test can be done with a given probability of success/failure. The estimation problem proceeds similarly. Note that the same methodology can be applied to derive a model on lower levels of knowledge, that is, for the follower function (equivalently, the boss and approval sets), the degrees, the influence graph and so on. It should be possible to test for example: "Is $S$ a boss set for agent $k$ ?" and so on.

In summary, the problem of identification of an influence model in a practical situation is a difficult one, and should initiate a new area of research.

\section{Future research on influence}

As one can conclude from this short overview, the influence issues create very complex problems that can be recognized in everyday life situations. Consequently, there are still many open questions that should be answered and many possibilities to continue the project on influence. We would like to finish the paper by mentioning some of our future research plans on these issues.

- The influence framework that we have studied so far is the one-step model, where a decision of an agent may be influenced by opinions of other players, but no possibility of iterating influence is assumed. In reality, the mutual influence does not stop necessarily after one step, as modeled by the influence framework studied by us so far, but may iterate. We intend to introduce dynamic aspects in the model, to study the behavior of the series of (different) influence functions, and to look for the convergence conditions for such series.

- We have compared our approach to influence based on social networks with the cooperative game theoretic approach based on command games. We are also going to compare the dynamic model of influence with the command games. In the framework of command games, an authority distribution over an organization was defined, and the power transition matrix of the organization was created. A Markov chain was used to describe the organization's long-run authority. In our future investigations, we could introduce the authority distribution based on the influence indices. 
- In order to measure influence between agents in a social network, we defined, in particular, the influence indices. However, we did not focus either on properties of these indices nor on their axiomatization. Consequently, in our future research on the influence topics, an axiomatic characterization of the influence indices should be provided.

- In our work on influence, we paid a lot of attention to the concept of influence function. In particular, we determined the exact relation between influence functions and follower functions. While we defined several influence functions and studied their properties, only deterministic functions were considered. Hence, it would be interesting to assume that the influence function is a probabilistic function. Such an assumption will model the reality in a more adequate way than restricting the analysis to deterministic functions.

- Our research on influence conducted so far was only theoretical. In order to get a deeper insight into the process of influence between agents, we would like to conduct some experiments on this issue, and to address the difficult problem of identification of the model, as presented in Section 5.

\section{References}

1. Banzhaf, J. (1965). Weighted voting doesn't work: a mathematical analysis. Rutgers Law Review, 19, 317-343.

2. Braham, M., \& Steffen, F. (2002). Voting power in games with abstentions. In M. J. Holler, H. Kliemt, D. Schmidtchen, \& M. E. Streit (Eds.), Power and Fairness (pp. 333-348). Mohr-Siebeck.

3. Brams, S. J. (1975). Game Theory and Politics. New York, Free Press.

4. Brams, S. J., \& Affuso, P. (1976). Power and size: a new paradox. Theory and Decision, 7, 29-56.

5. van den Brink, R., Rusinowska, A., Steffen, F. (2009). Measuring power and satisfaction in societies with opinion leaders: dictator and opinion leader properties. Submitted for publication.

6. Coleman, J. S. (1971). Control of collectivities and the power of a collectivity to act. In B. Lieberman (Ed.), Social Choice (pp. 269-300). New York: Gordon and Breach.

7. Coleman, J. S. (1986). Individual Interests and Collective Action: Selected Essays. Cambridge University Press.

8. DeMarzo, P. M. (1992). Coalitions, leadership, and social norms: The power of suggestion in games. Games and Economic Behavior, 4, 72-100.

9. Felsenthal, D., \& Machover, M. (1997). Ternary voting games. International Journal of Game Theory, 26, 335-351.

10. Felsenthal, D., \& Machover, M. (1998). The Measurement of Voting Power: Theory and Practice, Problems and Paradoxes. London: Edward Elgar Publishers.

11. Felsenthal, D., \& Machover, M. (2001). Models and reality: the curious case of the absent abstention. In M. J. Holler \& G. Owen (Ed.), Power Indices and Coalition Formation (pp. 87-103). Dordrecht: Kluwer.

12. Fischer, D., \& Schotter, A. (1978). The inevitability of the paradox of redistribution in the allocation of voting weights. Public Choice, 33, 49-67.

13. Grabisch, M., \& Roubens, M. (1999). An axiomatic approach to the concept of interaction among players in cooperative games. International Journal of Game Theory, $28,547-565$. 
14. Grabisch, M., \& Rusinowska, A. (2008). Influence functions, followers and command games. Submitted for publication.

15. Grabisch, M., \& Rusinowska, A. (2009a). Measuring influence in command games. Social Choice and Welfare, 33, 177-209.

16. Grabisch, M., \& Rusinowska, A. (2009b). A model of influence in a social network. Theory and Decision, Forthcoming.

17. Grabisch, M., \& Rusinowska, A. (2009c). A model of influence with a continuum of actions. Mimeo.

18. Grabisch, M., \& Rusinowska, A. (2009d). A model of influence with an ordered set of possible actions. Theory and Decision, Forthcoming.

19. Hoede, C., \& Bakker, R. (1982). A theory of decisional power. Journal of Mathematical Sociology, 8, 309-322.

20. Hojman, D., \& Szeidl, A. (2006). Endogenous networks, social games, and evolution. Games and Economic Behavior, 55, 112-130.

21. Holler, M. J., \& Packel, E. W. (1983). Power, luck and the right index. Journal of Economics, 43, 21-29.

22. Hu, X., \& Shapley, L. S. (2003). On authority distributions in organizations: controls. Games and Economic Behavior, 45, 153-170.

23. Hu, X., \& Shapley, L. S. (2003). On authority distributions in organizations: equilibrium. Games and Economic Behavior, 45, 132-152.

24. Isbell, J. R. (1958). A class of simple games. Duke Mathematical Journal, 25, 423-439.

25. Koller, D., \& Milch, B. (2003). Multi-agent influence diagrams for representing and solving games. Games and Economic Behavior, 45, 181-221.

26. König, T., \& Bräuninger, T. (1998). The inclusiveness of European decision rules. Journal of Theoretical Politics, 10, 125-142.

27. Laruelle, A., \& Valenciano, F. (2005). Assessing success and decisiveness in voting situations. Social Choice and Welfare, 24, 171-197.

28. Lopez-Pintado, D. (2008). Diffusion in complex social networks. Games and Economic Behavior, 62, 573-590.

29. Penrose, L. S. (1946). The elementary statistics of majority voting. Journal of the Royal Statistical Society, 109, 53-57.

30. Rae, D. (1969). Decision-rules and individual values in constitutional choice. American Political Science Review, 63, 40-56.

31. Rusinowska, A. (2008). On the not-preference-based Hoede-Bakker index. In L. Petrosjan, \& V. Mazalov (Eds.), Game Theory and Applications, 13, New York: Nova Science Publishers, Inc., Forthcoming.

32. Rusinowska, A. (2009). The Hoede-Bakker index modified to the Shapley-Shubik and Holler-Packel indices. Group Decision and Negotiation. Forthcoming.

33. Rusinowska, A., \& de Swart, H. (2006). Generalizing and modifying the Hoede-Bakker index. In H. de Swart, E. Orlowska, G. Schmidt, \& M. Roubens (Eds.), Theory and Applications of Relational Structures as Knowledge Instruments II LNAI 4342 (pp. 60-88). Heidelberg: Springer Verlag.

34. Rusinowska, A., \& de Swart, H. (2007). On some properties of the Hoede-Bakker index. Journal of Mathematical Sociology, 31, 267-293.

35. Schotter, A. (1982). The paradox of redistribution: Some theoretical and empirical results. In M. J. Holler (Ed.), Power, Voting, and Voting Power. Wurzburg-Wien: Physica-Verlag.

36. Shapley, L. S. (1994). A Boolean model of organization authority based on the theory of simple games. Mimeo.

37. Shapley, L.S., \& Shubik, M. (1954). A method for evaluating the distribution of power in a committee system. American Political Science Review, 48, 787-792.

38. Tchantcho, B., Diffo Lambo, L., Pongou, R., Mbama Engoulou, B. (2008). Voters' power in voting games with abstention: Influence relation and ordinal equivalence of power theories. Games and Economic Behavior, 64, 335-350.

39. van Winden, F. (2004). Interest group behavior and influence. In C. K. Rowley, \& F. Schneider (Eds.), The Encyclopedia of Public Choice (pp. 118-129). Springer. 